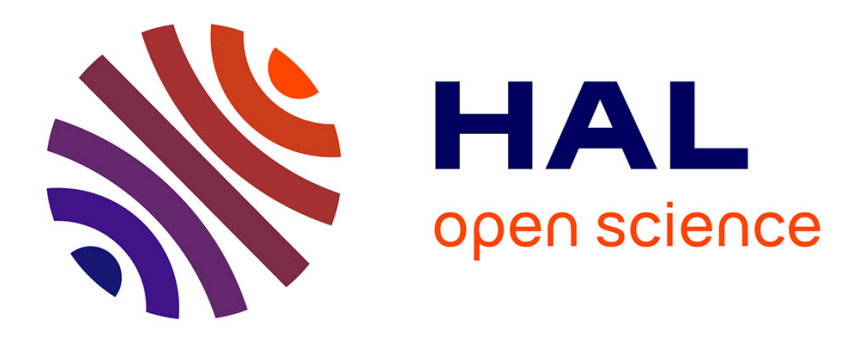

\title{
Conventions et comptabilité: vers une approche sociologique du modèle
}

Marc Amblard

\section{To cite this version:}

Marc Amblard. Conventions et comptabilité : vers une approche sociologique du modèle. Comptabilité

- Contrôle - Audit, 2004, numéro spécial, pp.47-68. halshs-00266967

\section{HAL Id: halshs-00266967 \\ https://shs.hal.science/halshs-00266967}

Submitted on 26 Mar 2008

HAL is a multi-disciplinary open access archive for the deposit and dissemination of scientific research documents, whether they are published or not. The documents may come from teaching and research institutions in France or abroad, or from public or private research centers.
L'archive ouverte pluridisciplinaire HAL, est destinée au dépôt et à la diffusion de documents scientifiques de niveau recherche, publiés ou non, émanant des établissements d'enseignement et de recherche français ou étrangers, des laboratoires publics ou privés. 


\section{Conventions et comptabilité : vers une approche sociologique du modèle}

Amblard M. (2004), «Conventions et comptabilité : vers une approche sociologique du modèle », Comptabilité, Contrôle, Audit, numéro spécial, juin, pp. 47-68.

\section{Marc Amblard}

\section{Résumé}

Une approche sociologique de la comptabilité permet d'enrichir sensiblement le modèle en faisant ressortir plus clairement qu'il met en mouvement un ensemble de règles et principes qui, loin de s'imposer d'eux-mêmes, résultent de choix collectifs à un moment donné. A cette fin, la théorie des conventions nous offre une grille de lecture pour le moins originale en retirant au producteur des comptes sa prétendue rationalité calculatrice pour le replacer dans des cadres convenus de coordination sociale et mimétique.

Mots clés. - théorie des conventions - incertitude norme - règle - comportement - conventions comptables.

\begin{abstract}
A sociological approach to accountancy improve the model because we understand the whole rules and principles which come from collectif choices at one moment. To this aim, convention's theory offers us an original reading. By this way, we awoid to counts' productor and we replace him in determined frames of mimetic and social coordination.
\end{abstract}

Keywords. - conventions theory - uncertainly proceedings - norm - rule - accounting conventions. 


\section{Introduction}

Sociologie et comptabilité : le rapprochement des deux disciplines pourrait presque paraître incongru tant les questionnements et les spéculations de la première tranchent nettement avec les équilibres et la rigueur de la seconde. Comment l'étude des phénomènes sociaux pourraitelle éclairer un système dont la principale finalité est d'enregistrer et communiquer un ensemble d'informations purement financières ${ }^{1}$ ? L'étonnement se dissipe toutefois rapidement et le rapport devient plus pertinent si l'on admet que le modèle comptable ne relève pas d'une vérité immanente mais repose sur une symbolique et des mécanismes qui résultent de choix humains à un moment donné. En ce sens, il s'agirait plus d'un construit progressivement pétri par les influences culturelles, les pressions sociales et les enjeux politiques. Le sens commun, reconnaissons-le, a trop souvent prêté à la comptabilité une neutralité à laquelle il lui est pourtant difficile de prétendre. Les évènements récents (Enron, Worldcom puis Parmalat ...) nous le rappellent assez brutalement. Le constat nous a alors incité à mener une réflexion quelque peu hétérodoxe en abordant le modèle selon un angle d'approche sociologique. A cette fin, la théorie des conventions, nous allons le voir, offre une grille de lecture des plus éclairantes.

Initié il y a quelques années, ce courant qui regroupe des recherches d'orientations diverses emprunte pour une grande part à la sociologie et dans une moindre mesure à la philosophie. Il fut mobilisé par les économistes avec pour principal objectif d'opposer une alternative enrichissante au courant contractualiste en remettant en cause ses hypothèses fondatrices. Selon les différents auteurs, c'est la notion de convention qui permet d'élargir l'analyse économique à des modes de coordination non marchands; dans le même temps, elle fait ressortir, à l'instar des sociologues ou anthropologues, la diversité des formes sociales qui expliquent les comportements humains telles les règles, les normes sociales, la culture, les valeurs, etc (Mercier, 2003).

Force est de reconnaître que la majeure partie de nos actes quotidiens qu'ils soient civils ou professionnels échappe le plus souvent à la rationalité calculatrice, s’inscrivant plutôt dans des cadres convenus de coordination mimétique et collective. En d'autres termes, l'individu peut échapper aux états d'incertitude consubstantiels à chaque situation non pas de façon autonome et souveraine mais en observant autour de lui les modalités d'accords, baptisés « conventions ». Ce faisant, qu'elles soient implicites ou explicites, les conventions constituent une réponse au chaos. Ciment d'une communauté, elles permettent de surmonter l'état de désorganisation en donnant à ses membres un ensemble de normes et valeurs communes. Du point de vue qui nous occupe, ce regard novateur invite alors à saisir que le producteur de comptes n'est pas un être suprême dont les seules qualités lui permettraient d'accéder à une solution optimale. Situation à choix multiples, la modélisation comptable est par nature source d'incertitudes; le professionnel échappe cependant à toute forme de blocage, les conventions coordonnant ses actes au sein d'un espace largement balisé (1). Un éclairage théorique permet alors de considérer la convention comptable comme un guide comportemental, faisant du modèle un corpus structuré qui met en mouvement un ensemble de règles et procédures socialement construites (2).

\section{La modélisation comptable : une situation à choix multiples}

Si la modélisation comptable consiste à produire la représentation chiffrée d'une entité économique, aucune solution ne s'impose dans l'absolu comme préférable à une autre. Aussi, devant la multiplicité des choix, le praticien serait en proie à l'incertitude s'il n'avait recours à 
un ensemble d'accords collectifs et reconnus. Ces conventions, en garantissant peu ou prou une convergence des pratiques assurent une coordination de l'information comptable (1.1.). Selon la nature des prescriptions qu'elles délivrent, il sera possible ensuite d'en proposer une typologie originale (1.2.). Pour éviter toute confusion, il conviendra enfin de positionner clairement la convention par rapport à la réglementation et au principe comptable (1.3.).

\subsection{De l’incertitude à la coordination}

Notion fondamentale dans la théorie des conventions, l'incertitude peut s'apparenter à une situation dans laquelle les facteurs qui influent sur l'action d'une personne en prise avec un problème existentiel ou pratique, ne sont pas tous déterminables ou prévisibles. Une solution consiste alors à conformer son comportement sur celui qu'on sait être communément admis dans ces conditions : la convention. Sa légitimité repose moins sur sa pertinence intrinsèque que sur son adoption généralisée : peu importe le sens de notre conduite, l'essentiel est que nous roulions tous dans le même sens. Ainsi, nous le verrons, la convention se présente comme un mode de coordination qui permet aux individus de résoudre des situations indécidables par leur seul calcul individuel.

\section{La modélisation comptable, source d' incertitude}

Commençons par mener notre réflexion en amont du processus. Se pose alors la question suivante: devant un événement particulier, le comptable se trouve-t-il en situation d'incertitude ? On serait tenté de répondre par la négative tant les automatismes qui l'animent sont nombreux et prégnants. Considérons le problème d'un peu plus près et nous constatons que notre praticien serait très rapidement embarrassé par la multiplicité des choix s’il n'avait recours à un cadre normalisé lui indiquant une solution attendue. Une première approche nous permet alors de recenser quatre sources principales d'incertitude.

- Première source d'incertitude : la délimitation du champ d'observation. Sans l'intervention des conventions comptables, une première série de questions viendrait à gagner l'esprit du praticien : de qui tient-on la comptabilité ? Où commence l'entreprise, où s'arrête-t-elle ? Quand doit-on arrêter les comptes ? Quels sont les événements qui relèvent de l'observation comptable ? Quels sont ceux qui en sont exclus et pourquoi ? Comment juger si une dépense est une charge ou une affectation du résultat? Etc.

- Deuxième source d'incertitude : le langage monétaire. Une seconde série de questions relatives à la façon de traduire et communiquer les flux repérés dans le champ d'observation, pourrait là encore plonger le praticien dans l'incertitude. Comment mesurer les flux qui naissent de l'activité de l'entreprise ? En d'autres termes, quel critère de mesure doit-on adopter? Comment donner une expression monétaire à certains événements qui relèvent notamment du non marchand ou du qualitatif ? Le comptable doit-il pour autant les ignorer ? Comment agréger des sommes exprimées à des époques différentes ? Etc.

- Troisième source d'incertitude : la procédure. Après avoir repéré le champ d’observation et le symbolisme utilisé, un certain nombre de questions se posent quant à la procédure à suivre : comment effectuer la saisie des informations sélectionnées (le mécanisme de la partie double ne s'impose pas de lui-même et n'a pas toujours prévalu) ? Quelle organisation comptable est la plus efficace ? Quels sont les documents obligatoires ? Comment orienter les comptes? Quelle présentation adopter ? Etc.

- Quatrième source d'incertitude : le fait générateur. Le moment exact qui va déclencher la procédure d'enregistrement est une source d'incertitude supplémentaire : à quel moment enregistre-t-on un flux ? Quand un bien doit-il ou peut-il être considéré comme une charge ? 
Comme un actif ? Doit-on enregistrer une charge seulement probable ? Peut-on enregistrer un produit de même nature ? Etc.

\section{La convention comme dispositif de coordination}

Cette liste est loin d'être exhaustive, et bien d'autres sources d'incertitude seraient à même de bloquer son comportement en plongeant le comptable dans la confusion et l'irrésolution.

Dans ces conditions, comment surmonte-t-il cette situation ? Peut-on lui prêter une autonomie de décision qui soit telle qu'il choisisse de façon isolée les règles idoines ? Est-il souverain au point de pouvoir émettre un jugement à chaque fois qu'une difficulté de cet ordre survient? Et même en postulant une rationalité parfaite, peut-on imaginer un seul instant que ses normes de références correspondront à celles des autres comptables ? Seront-elles acceptées par les utilisateurs de l'information comptable ? Rien n'est moins sûr.

On ne doit pas perdre de vue que la comptabilité est avant tout un système de représentation du monde économique qui répond à des objectifs de communication vers des utilisateurs en situations d'information limitée (Reix, 1995). Nos systèmes capitalistes s'accommodent difficilement d'une diversité des représentations comptables. Leur principale caractéristique est de s'appuyer sur une séparation entre l'épargne et la gestion des entreprises, le lien entre les deux étant assuré par la sphère financière. Cette disjonction requiert alors un langage commun, la comptabilité. C'est elle qui permet de comparer les entreprises entre elles et in fine de favoriser une allocation de l'épargne (Crouzet, Véron, 2002). Reflet de l'activité et de la situation économique d'une entreprise, la comptabilité est exprimée en unités monétaires selon des méthodes uniformes qui rendent ces données analogues et cohérentes d'une entreprise à l'autre. Ainsi, les conventions comptables découlent des exigences de comparabilité, de transparence et de permanence grâce auxquelles les décisions de financement peuvent se fonder sur des données financières.

Cependant, et malgré la communauté d'intérêt des individus, la coordination entre les acteurs du système comptable reste problématique du fait même de la multiplicité des solutions possibles. On l'a vu, aucune règle ne s'impose comme intrinsèquement préférable à une autre ; aussi a-t-il été nécessaire de " convenir » c'est-à-dire s’entendre sur les termes de la modélisation comptable ou si on préfère, construire des accords sur la façon de traduire des évènements économiques dans les livres comptables de l'entreprise.

C’est pourquoi, plutôt que de procéder à un calcul judicieux après une longue réflexion sur les conséquences de telle ou telle écriture, le comptable oriente ses actes en se référant à un ensemble de pratiques communément admises dans sa profession, les conventions comptables. Ces dernières étant le plus souvent confortées par une réglementation et un plan comptable assez complets. Grâce à elles, le professionnel agit la plupart du temps en toute quiétude ; sauf exception, il n'est jamais bloqué : il sélectionne dans son environnement les faits enregistrables, rejette les autres, les quantifie, les valorise, les convertit au besoin, arrête ses comptes à telle date et ceci dans le cadre d'une procédure administrative précise et acquise. A tel point d'ailleurs, qu'il en arrive à perdre conscience d'évoluer le plus souvent dans un espace convenu, c'est-à-dire résultant de choix à un moment donné. Les solutions s'imposent comme si elles étaient uniques. L'enquête que nous avons menée auprès d'un échantillon de professionnels du chiffre corrobore fortement ce point de vue. A titre d'illustration, nous en rapportons un extrait de six questions, toutes liées au processus d'activation comptable (présentation sommaire de l'enquête fournie en annexe) :

- Question n 14 : Connaissez-vous les raisons pour lesquelles les biens financés par créditbail ne figurent pas à l'actif?

- Question $n^{\circ} 15$ : Cela vous paraît-il logique?

- Question $n^{\circ} 20$ : Une entreprise fait l'acquisition d'un ordinateur à crédit, assortie d'une clause de réserve de propriété, doit-elle l'inscrire à l'actif du bilan? 
- Question $n^{\circ} 21$ : Existe-t-il une autre façon de procéder?

- Question $n^{\circ} 22$ : L'enregistrement actuel vous paraît-il logique?

- Question n 23 : N’y a-t-il pas contradiction avec l'enregistrement du crédit-bail ?

A la question $n^{\circ} 14$, une grande partie des praticiens (89\%) a répondu en avançant le principe de propriété. Quand nous leur avons demandé si cela leur paraissait logique (question $\left.n^{\circ} 15\right)$, ils ont été $46 \%$ à répondre par l'affirmative, $28 \%$ par la négative, le reste ayant des difficultés à prendre une position ferme. Ils sont en effet nombreux (plus de la moitié, en fait) à considérer que l'exclusion du bilan des biens financés par crédit-bail pourrait entraîner des déformations de l'image comptable selon le point de vue adopté.

La question $n^{\circ} 20$ relative à la clause de réserve de propriété ne présenta aucune difficulté aux professionnels interrogés qui répondirent dans une large majorité (89 \%) que le bien doit être inscrit à l'actif en dépit de la clause. A la question ( $\left.n^{\circ} 22\right)$ "l'enregistrement actuel vous paraît-il logique ? », ils ont été tout aussi nombreux à répondre par l'affirmative (92 \%). Il y a là une contradiction car les résultats obtenus montrent que près de la moitié des individus interrogés (46 \%) estime qu'il serait irrationnel d'immobiliser dans le bilan un bien financé par un dispositif (le crédit-bail) qui nous prive de sa propriété et, dans le même temps, logique d'inscrire à l'actif un ordinateur dont nous ne sommes pas plus propriétaires du fait de la clause qui en diffère le transfert.

\begin{tabular}{|l|c|c|c|c|}
\hline \multicolumn{1}{|c|}{ Questions } & oui & non & s.o. $^{1}$ & base \\
\hline $\begin{array}{l}\text { 15. Un bien financé par crédit-bail ne figure pas à l'actif du } \\
\text { bilan : cela vous paraît-il logique ? }\end{array}$ & 53 & 50 & 192 \\
\hline $\begin{array}{l}\text { 22. Un bien financé à crédit avec une clause de réserve de } \\
\text { propriété est tout de même inscrit à l'actif du bilan : cela vous }\end{array}$ & $92 \%$ & $3 \%$ & $26 \%$ & 6 \\
paraît-il logique? & $3 \%$ & $5 \%$ & 192 \\
\hline
\end{tabular}

Cette contradiction, somme toute partielle puisque ne concernant qu'une fraction des personnes interrogées (celles qui avaient répondu par l'affirmative à la question $n^{\circ} 15$ ), fut largement confirmée et étendue par les réponses recueillies à la question nº23.

\begin{tabular}{|l|c|c|c|c|}
\hline \multicolumn{1}{|c|}{ Questions } & oui & non & s.o. & base \\
\hline $\begin{array}{l}\text { 23. N'y a-t-il pas contradiction, selon vous, entre les deux } \\
\text { enregistrements? }\end{array}$ & 19 & 146 & 13 & 178 \\
\hline
\end{tabular}

Près de $82 \%$ des professionnels ont en effet répondu spontanément qu'ils ne voyaient aucune contradiction dans le fait qu'un bien financé par un contrat de crédit-bail soit exclu du bilan et qu'inversement, un bien y soit inscrit malgré la clause qui vient en frapper l'acquisition. Il est alors piquant de constater que les personnes qui émettaient des doutes quant au bien fondé de l'enregistrement comptable du crédit-bail, donc conscientes des limites de la stricte application du principe de propriété, n’ont pas détecté de prime abord l'antinomie des deux enregistrements.

Comme toute autre convention, les conventions comptables dotent le praticien d'un ensemble de mécanismes qui le soustraient aux multiples situations d'incertitude. Toutefois, la légitimité d'un enregistrement représente en fait beaucoup moins l'aboutissement d'un processus individuel de réflexion rationnelle que la reconnaissance et l'anticipation (pas forcément perçue) d'une acceptation collective. De surcroît, les conventions sont à ce point

\footnotetext{
${ }^{1}$ Sans opinion
} 
prégnantes que les praticiens concernés en oublient leurs fondements et perdent à leur égard une partie du sens critique. Peu à peu, celles-ci s'imposent comme des évidences indiscutables.

\subsection{Esquisse d'une typologie}

De cette réflexion, il ressort que les travaux du comptable ne sont pas le résultat de choix individuels. Celui-ci serait constamment en proie à l'incertitude s’il ne s'appuyait sur un ensemble de procédures collectives préétablies. En ce sens, la convention comptable peut être appréhendée comme un transmetteur d'informations qui coordonne le comportement du professionnel en "balisant » sa tâche par un ensemble de repères. Il est alors possible d'en dresser une typologie en fonction de la nature du « discours » délivré au sujet. Quatre grandes catégories se distinguent: les conventions d'observation, les conventions de mesure, les conventions de réalisation et les conventions de procédure.

\section{Les conventions d'observation}

Les conventions d'observation ont pour rôle de délimiter le champ d'observation du comptable en dessinant les frontières de l'entité comptée. Il est alors possible d'en faire une représentation comptable autonome. Le principe d'entité a permis d'émanciper l'entreprise, tant en ce qui concerne les structures individuelles que les entreprises sociétaires ${ }^{2}$. Aussi, après l'avoir détachée de ses propriétaires, convient-il d'en préciser les contours : quel est l'objet dont on veut produire une représentation comptable ? Où commence-t-il ? Où s'arrêtet-il ? La convention de patrimonialité est alors fortement prédominante dans ce processus de bornage. Encore faudrait-il préciser à ce sujet que nous ne partageons pas tout à fait la même approche que les anglo-saxons, eux-mêmes ayant convenu d'autres critères pour tracer le périmètre de l'objet compté. Les incidences sur la représentation ne sont pas négligeables. L'enregistrement du crédit-bail en est un exemple.

Situer l'entreprise dans l'espace ne suffit pas, le comptable doit aussi intégrer la dimension du temps dans sa phase d'observation. A moins d'attendre la dissolution de l'entreprise, ce qui n'est guère envisageable, un découpage du temps s'impose pour apprécier à intervalles réguliers le patrimoine et les performances de l'entreprise ; la période d'observation étant généralement égale à un an, nous parlons de convention d'annualité.

\section{Les conventions de mesure}

L'entreprise observée étant «bornée » dans le temps et dans l'espace, il est nécessaire de distinguer les flux qui relèvent de l'observation comptable de ceux qui en sont exclus. Il importe ensuite d'en prendre la mesure. Deux conventions s'imposent alors : la convention de quantification monétaire et la convention des coûts historiques. La première signale au comptable que les flux et les stocks relevant de l'observation comptable doivent être estimés en unités monétaires. Il va sans dire que cette convention écarte du domaine comptable un certain nombre d'événements non-marchands, difficilement estimables en monnaie.

Quant à la seconde, elle indique au praticien la technique d'évaluation retenue. Il existe de nombreuses méthodes pour évaluer le prix d'un bien: valeur historique, actuarielle, de marché ... ; ne répondant pas au même objectif, aucune n’est d'application générale. Aussi, pour garantir à la comptabilité sa comparabilité, a-t-il fallu « convenir » d'un critère unique d'évaluation : le coût historique. Signalons à ce sujet le passage dans quelques mois à une nouvelle convention, la juste valeur, concept anglo-saxon introduit par les normes IFRS (International financial reporting standards) à destination des sociétés cotées. 
Enfin, une troisième convention de mesure, la convention de continuité, participe au processus de modélisation comptable en indiquant que les comptes doivent être tenus en présumant que l'entreprise continuera indéfiniment son exploitation.

\section{Les conventions de réalisation}

Mesurer le revenu de l'entreprise suppose que l'on ait fixé préalablement des conventions quant aux conditions de sa réalisation. Il est en effet nécessaire de définir la nature de ce revenu et d'indiquer le moment de sa constatation effective. Quatre conventions importantes dissipent alors toute incertitude. La convention de qualification permet de repérer dans un flux, une charge ou un produit. Cette qualification n'a rien d'évident : pour quelle raison, la rémunération des apporteurs de capitaux serait-elle qualifiée de simple dépense d'affectation (du résultat) et non de charge (Gensse, 1995) ? Dans ce cas, pourquoi ne pas considérer la rémunération des autres partenaires (personnel, prêteurs...) comme des flux de répartition plutôt que de les enregistrer en charges ? Là encore, rien n'est donné tout est convenu. De son côté, la convention de reconnaissance indique que les produits sont comptabilisés lorsqu'ils sont acquis et les charges lorsqu'elles sont engagées juridiquement. De fait, le résultat d'une période ne correspond nullement à l'encaissement net perçu par l'entreprise mais à un revenu « en attente » ou potentiel. Pour sa part, la convention de rattachement indique au comptable de rattacher les charges (de période) à l'exercice au cours duquel les produits correspondants sont constatés. Enfin, la convention de prudence prescrit une appréciation raisonnable voire pessimiste des faits observés ; son respect permet d'éviter le risque de transfert sur l'avenir d’incertitudes présentes susceptibles de grever le patrimoine et les résultats de l'entreprise.

\section{Les conventions de procédure}

L'activité de l'entreprise se traduisant par des échanges et des transactions avec d'autres unités, la comptabilité a pour fonction de relever et saisir les événements qui modifient la consistance de son patrimoine. Il est donc nécessaire d'adopter une procédure spécifique faisant appel notamment à un instrument : le compte. Les comptes sont reliés entre eux par le mécanisme de la partie double au sein d'un système ayant pour finalité la production périodique des états financiers. Deux conventions viennent informer le praticien, la convention de partie double et la convention de présentation. La première fixe le fonctionnement et la coordination des différents comptes utilisés par l'entreprise et la seconde indique la façon dont il doit présenter les documents comptables. La présentation est parfois normalisée et obligatoire comme en France, mais elle peut ne pas l'être, comme aux EtatsUnis, par exemple ; dans ce dernier cas, une grande majorité d’entreprises a néanmoins choisi de produire des documents semblables, toutes ayant compris qu'il était dans leur intérêt d'adopter la même convention de présentation : la comparabilité des comptes de sociétés et la mise en confiance des utilisateurs passent en effet par l'uniformisation des présentations.

La comptabilité repose ainsi sur une multitude de conventions dont les différents discours viennent guider le praticien en lui indiquant les actes "normaux » c'est-à-dire ceux qu'on est normalement en droit d'attendre de lui. C'est pourquoi chaque praticien est informé des critères de choix retenus par la collectivité. Il sait ce que les autres font, ce qu'il doit et ce qu'il ne doit pas faire. Cela dit, il n'est pas dans l'objet du discours de la convention de révéler à l'individu la solution idéale à un problème rencontré, mais plutôt d'indiquer ce qui est collectivement bon, c'est-à-dire ce que chacun croît être bon. La nuance est de taille. Pour cette raison, le respect de ces conventions n'assure pas à l'utilisateur de l'information comptable une représentation parfaite de la réalité : elles contribuent à dessiner une image qui en est nécessairement une schématisation et donc une réduction. 


\subsection{Réglementation, principes et conventions}

Malgré cette différenciation typologique, les conventions tendent à s'agréger pour former un corpus cohérent dont le rôle consiste à borner les actes du comptable, en lui indiquant ce qu'il est " convenu » de pratiquer dans chaque situation. Pour autant, il serait erroné de confondre convention et réglementation comptable ; on serait tenté de penser que le droit comptable avec ses lois et ses règlements contraint les comportements en imposant des normes. La réalité est cependant plus nuancée et plus complexe : la loi se contente souvent d'entériner une convention préexistante. Le fait n'est pas nouveau, les philosophes ont toujours soutenu l'idée que le droit est né quelques jours après le monde. La législation n'est qu'un moyen parmi d'autres de porter à la connaissance des citoyens, le discours des conventions. Elle en représente généralement, la traduction écrite et participe à leur transmission en véhiculant le message conventionnel auprès de l'utilisateur du système. Aussi, une loi ne s'imposera réellement qu'à la condition d'emporter l'adhésion du plus grand nombre. Le praticien sera d'autant plus déterminé que l'adoption sera généralisée. Nombre de lois sont tombées dans l'oubli faute d'avoir durablement convaincu la population. Il existe entre les deux concepts une subtile relation : la convention engendre la loi lorsqu'il convient de contenir et sanctionner formellement les déviances mais, dans le même temps, le droit renforce la convention en alimentant la croyance que tous s'y conforment. C'est ainsi que la normalisation comptable, en établissant des règles communes, participe à la convergence des pratiques et amplifie simultanément la conviction des praticiens dans une adoption généralisée des conventions.

Au même titre qu'on ne peut l'identifier à une prescription réglementaire, on ne saurait non plus confondre convention et principe comptable. Bien que les professionnels aient donné le nom de principes à une grande partie des conventions, on ne peut assimiler systématiquement les deux notions. Si une grande partie des principes relèvent d'une approche déductive de la comptabilité (ils sont nés de la pratique) et ont valeur de convention, d'autres sont plutôt le fruit d'une tentative de normalisation et s'en différentient sans équivoque.

Prenons l'exemple du principe d'image fidèle, plusieurs fois repris dans les articles de la quatrième directive européenne et dans la loi comptable française : "Les comptes annuels doivent être réguliers, sincères et donner une image fidèle du patrimoine, de la situation financière et du résultat de l'entreprise ». Le texte précise même que si l'application d'une prescription comptable se révèle impropre à donner une image fidèle de l'entreprise, il doit y être dérogé. Pourtant, de nombreux cas témoignent de son inapplication et ce pour plusieurs raisons ; en tout premier lieu, il faudrait préciser ce qu'est une image fidèle et surtout à quelle réalité les comptes doivent être fidèles. A cela certains auteurs comme Colasse (1996, p. 30) objecteront que "l'entreprise n'est pas une réalité qui s'impose toute seule, c'est un construit : ce n'est pas un fait, elle est, au moins en partie, faite par son observateur »; on pourrait, au demeurant avancer qu'il y a autant d'images fidèles de l'entreprise que d'observateurs ou d'utilisateurs de l'information comptable. Deuxième raison concernant plus directement notre propos, l'application du principe d'image fidèle se heurte, du moins en France, au respect de certaines conventions: pouvons-nous soutenir que l'adoption simultanée de la convention des coûts historiques et la convention de prudence contribue à donner une image fidèle de l'entreprise ? Fidèle à la réalité économique ? On peut en douter lorsqu'un bilan contient par exemple des immeubles existants mais totalement amortis ou lorsqu'un portefeuille de SICAV monétaires figure à l'actif depuis plusieurs années pour sa valeur d'acquisition. Les exemples ne sont pas rares, ils illustrent simplement l'inanité d'une recommandation, d'un principe ou d'un texte qui va à l'encontre de certaines conventions. Toujours pour marquer la distinction entre convention comptable et principe comptable, 
signalons à l'inverse que le comportement des praticiens repose sur des conventions particulières qui n'ont pourtant jamais donné naissance à des principes reconnus. Il en va ainsi pour certaines conventions d'observation dont on ne trouve aucune trace dans notre cadre conceptuel ; c'est le cas notamment de celles qui permettent au comptable de fixer les frontières de l'entité dont il doit rendre les comptes : où commence-t-elle ? Où s'arrête-t-elle ? Dans quelle mesure doit-elle être distinguée de ses actionnaires ? Quels sont les événements qui l'animent? Quels sont ceux qui sont exclus de la représentation ? Force est d'avouer que les cadres normalisés sont très discrets à ce sujet. Ainsi, si certaines conventions sont devenues des "principes comptables généralement reconnus" (coûts historiques, prudence...), d'autres en revanche n'ont jamais fait l'objet d'une reconnaissance identique (observation, qualification...). Il n'en demeure pas moins qu'elles guident subtilement les travaux du professionnel en dictant ce que sa communauté reconnaît comme solutions admises.

\section{La convention comptable : un guide comportemental}

La première partie nous a invités à considérer la comptabilité comme un ensemble de conventions implicites et explicites coordonnant les agents dans la résolution des différents problèmes de représentation comptable. La théorie des conventions devrait nous permettre d'enrichir le modèle en mobilisant son arsenal de concepts et raisonnements. Commençons alors par replacer cette jeune théorie en quête de légitimité scientifique dans le contexte économique qui lui a permis de devenir un courant de pensée à part entière (2.1). L'application d'une axiomatique conventionnaliste précise viendra ensuite soutenir et renforcer la démonstration (2.2.) avant de présenter les principales caractéristiques des conventions comptables (2.3.).

\subsection{Positionnement théorique}

Appelée aussi " économie des conventions ", cette recherche s’est développée en se nourrissant des reproches couramment adressés à l'encontre de la théorie contractualiste de l'entreprise. Celle-ci postule que toute relation socio-économique peut se manifester dans des contrats passés entre les individus. Ce sont ces contrats qui guident le comportement des acteurs, lesquels cherchent à optimiser leur intérêt individuel. Les théories des coûts de transaction, de l'agence et des droits de propriété ont donné à ce modèle une solide assise conceptuelle, lui permettant ainsi d'alimenter abondamment les disciplines de gestion. Cependant, certaines questions restent sans réponse, et progressivement de nombreuses critiques s'élèvent donnant ainsi naissance à de nouvelles hypothèses: les échanges représenteraient une structure collective et non le résultat du libre jeu des acteurs. Les relations économiques reposent certes sur des rapports de calcul, mais également de confiance et d'honneur. Bien que négligées par le modèle classique, les croyances et les représentations y joueraient même un rôle très important.

Les différentes recherches mettent alors en évidence plusieurs formes d'arrangements internes susceptibles d'expliquer comment l'action collective dans l'entreprise est capable de dépasser les divergences pour s'ordonner autour d'un projet fédérateur. Le fonctionnement des entreprises, nous expliquent les sociologues Boltanski et Thévenot (1983), ne s’appuie pas seulement sur l'appartenance à une culture commune ou sur l'agrégation de stratégies individuelles mais sur un certain nombre de conventions passées entre les individus ou les groupes. L'entreprise est présentée comme un lieu de tension naturelle entre des logiques 
contradictoires que les individus s'attachent à réduire au moyen de " normes de conduite ». En effet, selon les auteurs, les acteurs ont une capacité commune à se référer à des principes de justice considérés comme légitimes et à l'aide desquels ils justifient leur action. Les accords sont possibles grâce à la construction d'un état de " généralité », lors " d'épreuves » où chacun essaie d'intégrer la logique de l'autre. Autrement dit, le calcul des agents est largement conditionné par des contingences sociales qui les dominent.

Ainsi, de nombreux travaux de recherche se mobilisent, articulant perspectives sociologiques et économiques pour dégager de proche en proche un courant de pensée qui, à défaut d'être convergent et homogène, mobilise les mêmes points de vue quant aux moteurs de l'action collective. Une théorie voit alors le jour à la fin des années quatre-vingts : la théorie des conventions. Il n'y est nullement question de rejeter le contrat et le calcul mais de s'attarder sur la constitution des référentiels d'action collective qui les surplombent ; en d'autres termes, élever au rang d'objet de la science économique les modes de construction et de mobilisation des règles collectives afin de comprendre les mécanismes de coordination (Gomez, 1995). En contrepoint des approches classiques, ce sont les représentations collectives qui servent de présupposé commun à l'action (Thévenot, 1989) et permettent une certaine coordination $a$ priori des anticipations individuelles (Orléan, 1989). L'élément fondateur du courant théorique émerge assez clairement : l'accord entre les individus, même lorsqu'il se limite au contrat marchand, n'est pas possible sans un cadre commun, une convention constitutive (Dupuy et alii, 1989). Aussi les formes collectives de la coordination occupent-elles toujours une place centrale dans les analyses conventionnalistes et apparaissent, selon les auteurs, sous la forme de " contextes communs d'interprétation » (Salais, 1994), ou encore de " dispositifs cognitifs collectifs » (Favereau, 1989). Pour l'essentiel, la réflexion porte alors sur les mécanismes de construction, de stabilisation et d'évolution de ces formes conventionnelles (Detchessahar, 2003).

En somme, comme le souligne Dupuy (1989), nous assistons à un glissement du paradigme de l'Homo oeconomicus vers celui d'Homo sociologicus : le premier est dégagé de tout ce qui fait la vie en société : influences, imitations, soumission, déterminisme social ; il est autonome et souverain ; sa rationalité lui permet de maximiser son bien-être en exploitant les moyens rares dont il dispose. Le second a perdu son autonomie, guidé par des normes, coutumes, règles qu'il a progressivement intégrées depuis son plus jeune âge et jusque dans l'organisation qui l'accueille : «Celles-ci, automatiquement, mécaniquement, le déterminent à agir, fut-ce au détriment de son intérêt bien compris. » (Dupuy, 1989, p. 361).

\subsection{Validation axiomatique}

A ce stade de la réflexion, la théorie des conventions va nous offrir la possibilité d'affermir notre position en vérifiant l'hypothèse centrale de ce travail selon laquelle l'acte comptable s'inscrit dans un cadre conventionnel. Nous décidons alors de recourir au mode opérationnel de repérage développé par Lewis (1969). Pour ce philosophe et logicien, la convention est une "régularité dans le comportement " qui s'élève comme un dispositif permettant aux individus placés dans une situation récurrente de résoudre un problème de coordination. Résultat d'une interaction stratégique, elle est modélisée avec le langage de la théorie des jeux (Gensse, 2003). Sa contribution au courant conventionnaliste est aujourd'hui largement reconnue par les différents auteurs, notamment les économistes auprès desquels il apparaît comme la référence obligée (Batifoulier, Larquier (de), 2001). On lui sait gré, entre autres, d'avoir développé dans son ouvrage de philosophie une méthode axiomatique destinée à repérer et identifier les caractéristiques d'une convention. Il en ressort dans le même temps une définition précise du concept auxquels se sont ralliés nombre de conventionnalistes 
français comme Dupuy (1989), Orléan (1994) ou Gomez (1996). Notre étude des conventions comptables prendra justement appui sur les cinq propositions de Lewis que nous considèrerons comme autant de tests à valider sur le terrain comptable.

Proposition 1 - Chacun se conforme à la convention - La convention comptable, par son message a permis au praticien de repérer le comportement adopté par l'ensemble de la collectivité. L’appartenance à un corps professionnel et la reconnaissance de la compétence supposent que chacun adopte la convention. Comprenons bien que l'adoption des conventions comptables élève le praticien et plus généralement l'utilisateur au statut de membre : en maîtrisant progressivement le langage institutionnel qu'est la comptabilité, il s'affilie à un groupe, intègre une communauté. Une fois assimilé, le membre n'a pas à s'interroger sur ses actes: les règles explicites et implicites qu'il a progressivement enregistrées guident sa conduite. A l'inverse, la dissidence conventionnelle aurait pour effet d'exclure l'individu, remettant ainsi en cause sa fonction professionnelle et sociale. Notre enquête a d'ailleurs révélé une absence de déviances par rapport à la régularité des pratiques. Ainsi, les personnes interrogées ont massivement jugé (98 \%) que leur conduite était fidèle aux usages de la profession. Certes, rien ne prouve leur bonne foi, mais à la réflexion, ce résultat n’a rien d'étonnant pour celui qui a pratiqué la comptabilité en cabinet ou en entreprise : l'acteur se réfère aux conventions par réflexe et par intérêt, la conformité au nombre constituant à ses yeux la meilleure légitimité. Cela ne signifie aucunement que les comportements observés sont totalement respectueux des prescriptions réglementaires, nous ne parlons pas de droit mais de pratiques observées, ce qui est très différent. La question d'ailleurs, ne faisait absolument pas référence au droit comptable mais aux usages. Ainsi, l'exemple de l'amortissement dérogatoire illustre assez bien l'écart entre la norme en tant que prescription réglementaire et la norme au sens de régularité statistique. Dans la quasi-totalité des cas (90\% des personnes interrogées, question $n^{\circ} 26$ ), l'enregistrement d'un amortissement dégressif reconnu ne pas correspondre à l'amortissement économiquement justifié, ne donne lieu à aucun calcul d'amortissement dérogatoire en dépit de l'obligation. Même si les praticiens admettent un manquement à la règle prescrite par la loi comptable, ils sont aussi conscients, on va le voir, que leur comportement est en phase avec celui de leurs pairs. Dès lors, cette connaissance commune peut constituer une justification et une protection autrement plus efficace que le simple respect des règles légales.

Proposition 2 - Chacun anticipe que tous se conforment à la convention - Le comptable anticipe que toute la population adopte la convention; nous venons de le préciser, chaque comptable justifie ses actes et se met à l'abri en précisant qu'ils sont conformes aux pratiques du milieu. Il limite de cette façon sa responsabilité en cas de litige, mais dans le même temps renforce la convention en montrant aux autres qu'il l'adopte. Ainsi, à la question $n^{\circ} 2$ : " Quel est votre sentiment à l'égard de vos confrères : pensez-vous qu'eux aussi se conforment aux usages de la profession? ", les praticiens interrogés ont répondu par l'affirmative dans $87 \%$ des cas et seuls $2 \%$ ont apporté une réponse opposée. Nous retrouvons là, la conscience que chacun a de la conformité de son comportement à ceux adoptés par ses pairs sur un territoire donné.

Proposition 3 - Chacun préfère une conformité générale à la convention - Toute déviance serait considérée comme une entrave à l'unanimité et remettrait en cause la pertinence de l'enregistrement comptable. Celui-ci n'est pas juste en soi, et peu importe qu'il le soit, le principal étant que tous s'y conforment ; au sein du raisonnement conventionnaliste, c'est justement la préférence généralisée pour une solution donnée qui lui confère un sens et une justification. Afin de valider la proposition, nous avons inclus dans le sondage la question 
suivante $\left(\mathrm{n}^{\circ} 4\right)$ : "Préférez-vous que tous s’y conforment (aux usages comptables) dans le cadre d'une pratique homogène ou bien êtes-vous indifférent à une multiplication des déviances qui aboutirait à une pratique hétérogène ? ". Les résultats ont alors montré que chaque professionnel interrogé n’est pas indifférent aux pratiques de ses confrères puisque près de 90 \% de notre échantillon s'accordent à considérer qu'une pratique homogène de la comptabilité est préférable; seuls $5 \%$ sont indifférents aux pratiques exercées par leurs confrères. Il est ainsi apparu qu'une conduite divergente pouvait mettre le professionnel dans l'embarras; il nous a semblé, mais les réponses obtenues n'ont pas permis de confirmer clairement cette impression, que l'embarras résulte moins de l'atteinte au principe de comparabilité des comptabilités, que de la remise en cause de la règle supposée acquise. En d'autres termes, un comportement divergent est réellement gênant en ce qu'il contredit indirectement la régularité et la généralisation de la pratique adoptée, et partant, l’anticipation de ce que les autres font.

Proposition 4 - Il existe au moins une alternative à la convention comptable - La convention n'est pas unique, une autre convention peut lui être opposée. La règle comptable retenue par la profession est une solution parmi d'autres. Le principe de la partie double par exemple n'est pas incontournable, diverses études l'ont montré et il est possible qu'on assiste un jour à sa remise en cause et son remplacement par un autre mécanisme plus performant. De même, le principe des coûts historiques est encore une méthode convenue et d'autres méthodes toutes aussi valables pourraient s'y substituer : prix d'acquisition actuel, prix de revente, valeur actuelle des revenus, etc. La réforme en matière de juste valeur, nous l'avons dit, est suffisamment éloquente à cet égard. Aucun de ces principes n'est valable dans l'absolu, ils ne répondent pas aux mêmes exigences. Nous pourrions continuer et montrer que toute solution choisie admet au moins une alternative ; par conséquent, toute convention peut être remise en cause par celle-ci.

La présence d'une solution alternative nous renvoie alors aux fondements mêmes de la théorie en ce qu'elle est génératrice de l'incertitude décrite dans la première partie : c'est bien de la multiplicité des solutions choisies que naît l'indécision de l'agent en l'absence de convention. Le sondage est ici inopérant attendu que le repérage de l'alternative relève plus d'une analyse conceptuelle de la convention comptable que d'une interrogation du convenant, souvent peu conscient du caractère alternatif de la convention.

Proposition 5 - Les quatre propositions précédentes forment un « Common Knowledge » En d'autres termes, chacun les connaît, sait que les autres les connaissent de la même manière et que chacun sait que l'autre sait aussi... Certes, un tel concept laisse supposer une boucle spéculaire infinie peu réaliste, mais Lewis lui-même reconnaît que la coordination conventionnelle se contente d'un nombre limité d'anticipations. Quelques années avant lui, Schelling avançait déjà que la coordination des individus était rendue possible grâce à la connaissance et la prédiction des intentions d'autrui. Plus tard, Dupuy (1989) souligna à son tour l'importance de la spécularité dans la régularité des comportements, tout en relevant les paradoxes qu'elle peut susciter.

Concernant notre étude, cette connaissance commune a pu être vérifiée, tout au moins pour les trois premières sous-propositions.

- Sous-proposition 5-1: chacun sait que globalement tous se conforment aux conventions (et sait que chacun sait ...). Il suffit de revenir un instant aux résultats issus de la question $n^{\circ} 2$ pour admettre l'aspect spéculaire de la conformité générale aux conventions comptables : «Quel est votre sentiment à l'égard de vos confrères : pensezvous qu'eux aussi se conforment aux usages de la profession? » (taux de réponses positives : $87 \%)$. 
- Sous-proposition 5-2: chacun sait que chaque praticien anticipe que tous se conforment à la convention. Nous avons alors posé la question suivante $\left(n^{\circ} 3\right)$ : « Pensezvous que vos confrères partagent votre sentiment ? En d'autres termes, pensez-vous que les autres praticiens prêtent à leurs homologues la même conformité aux usages de la profession? ». Par l'effet de miroir sur lequel elle s'appuie, la question est délicate et nécessita en conséquence quelques explications avant d'obtenir une réponse. Une bonne majorité des praticiens (61\%) a alors répondu positivement ; 5 \% d'entre eux ont apporté une réponse négative et $36 \%$ ont préféré s’abstenir.

- Sous-proposition 5-3 : chacun sait que chacun préfère une conformité générale à la convention. Pour tester cette proposition, nous avons fait suivre la question $n^{\circ} 4$ («Préférez-vous que tous s’y conforme ...») d'une question annexe : "Selon vous, comment les autres praticiens interrogés ont-ils répondu à la question précédente ? »; 77 \% des praticiens interrogés ont alors estimé que leurs homologues avaient dû répondre positivement à la première question. Cela signifie qu'une large majorité pense que les autres praticiens préfèrent une conformité générale à une multiplication des déviances. Uniquement $5 \%$ des personnes interrogées ont pensé le contraire.

- Sous-proposition 5-4 : chacun sait qu'il existe au moins une alternative à la convention. Force est de reconnaître en revanche, qu'il nous est difficile de valider cette proposition. En effet, nos observations in situ ont montré que les pratiques sont à ce point intériorisées, que les convenants ont perdu toute trace de leur justification originelle et de leur caractère alternatif. Ainsi, parmi les praticiens interrogés (question $n^{\circ} 16$ ), un peu plus d'un tiers reconnaissent par exemple, que d'autres critères que la propriété juridique pourraient être utilisés en tant que principe d'inclusion dans le périmètre de l'entité comptable ; de même, il n’y eut que $4 \%$ des personnes interrogées (question $n^{\circ} 31$ ) à considérer qu'il peut exister d'autres façons d'enregistrer les écarts de conversion. En revanche, ils sont plus de $80 \%$ à considérer qu'il existe d'autres critères d'évaluation que celui des coûts historiques (question $n^{\circ} 33$ ).

Il convient de signaler, néanmoins, que l'aspect « Common Knowledge » des conventions est loin de faire l'unanimité chez les conventionnalistes, et notamment à l'égard de la quatrième proposition concernant l'alternative. Ainsi, Dumouchel (1992, p. 18) montre-til à ce sujet, que la notion de convention ou règle chez certains économistes comme Hayek diffère sensiblement de la convention chez D. K. Lewis : " même si les membres de $\mathrm{P}$ (la population) observent $\mathrm{R}$ (la convention au sens de régularité) parce que tous les autres le font, qu'ils s'attendent à ce que presque tous les autres le fassent, et savent que c'est pourquoi ils observent $R$, ils n'ont généralement pas en tête une régularité de remplacement $\mathrm{R}^{\prime}$ et ne savent généralement pas que si presque tous se conformaient à $\mathrm{R}$ ' plutôt qu'à $\mathrm{R}$, presque tous préféreraient qu'au moins une personne de plus se conforme à R' ». Il semble, pour notre part, que c'est justement le propre d'une convention que de guider l'individu dans la résolution de problème, jusqu'à faire disparaître le problème luimême, de sorte qu'aucune question ne se pose et que la voie à suivre s'ordonne comme une évidence. Nous rejoignons alors l'avis de Favereau (1989) qui précise que les règles s'imposent aux personnes dans l'instant courant, comme des présupposés dont les conditions sociales de la genèse sont oubliées. Progressivement, l'adhésion aux conventions s'immerge dans une opacité à l'intérieur de laquelle les individus n'ont pas conscience que les règles qu'ils suivent pourraient être évaluées et comparées à d'autres règles possibles (Orléan, 1989).

Pour ces raisons, nous décidons de passer outre la non-validation de la sous-proposition $\mathrm{H}$ 5-4 et estimons pouvoir qualifier la règle comptable de convention sans risquer d'en dévoyer le concept. Nous en donnons alors la définition suivante : la convention comptable est un 
ensemble de procédures cognitives préétablies, permettant aux agents de résoudre des problèmes de représentation comptable en guidant leurs pratiques dans un espace normé.

\subsection{Etude organique}

La reconnaissance axiomatique des conventions qui fondent le modèle comptable nous invite maintenant à en examiner plus précisément les principales caractéristiques.

- Chacune d'entre elles se caractérise par un « espace » à l'intérieur duquel elle emporte la conviction des adopteurs; déplaçons-nous d'un espace à un autre, les conventions changent ; les choix n'ont alors de justification que par rapport à celles qui leur servent de référentiel. C’est le principe de territorialité des conventions. Ainsi, dans tel secteur les indemnités de départ à la retraite seront systématiquement provisionnées alors qu’il y sera fait totalement abstraction dans tel autre secteur. "Toute vérité est vérité d’un élément, d'une heure et d'un lieu ... » (Deleuze, 1988, p. 125).

- De plus, c'est l'adhésion de l'ensemble de la profession qui permet à la convention comptable d'exister. Chaque praticien sait aussi qu'elle est respectée par ses pairs ; peu importe qu'elle soit juste ou non, que l'image qu'elle produit soit fidèle ou non, le plus important étant que son adoption par la collectivité puisse fournir une justification à son acte; c'est ainsi qu'il rationalise son comportement. En tant que procédure de représentation collective, sorte de langage commun, la comptabilité ne présenterait que peu d'intérêt si elle n'emportait l'adhésion de tous ; la fonction sociale de la comptabilité ne consiste-t-elle pas justement à produire une croyance collective ? Certains auteurs l'ont d'ailleurs fort bien exprimé : "le problème n’est pas d'apporter une vérité comptable objective qui engendrerait la confiance comme le pensent la plupart des praticiens et de nombreux auteurs comptables, mais d'apporter une croyance, un bien commun, une référence commune qui permettrait aux principaux acteurs et à un moment donné, de dialoguer, de s’affronter, d’effectuer des transactions, de négocier... » (Capron, 1990).

- Ainsi une convention comptable ne s'impose pas ; on ne l'adopte pas parce qu'elle est obligatoire, mais répétons-le, parce qu'elle suscite l'adhésion d'une population suffisamment vaste pour s'imposer comme la norme. De fait, chaque praticien trouve normal de s'y référer. La tentation est grande, à ce sujet, d'expliquer le respect des conventions par la crainte du châtiment. Il est indiscutable que l'intérêt bien compris de l'acteur peut parfois l'amener à considérer les risques de sanction civile ou pénale dans ses calculs ; cependant, si notable que puisse être ce facteur, il ne saurait à lui seul rendre compte de la conformité. A cet égard, de nombreux sociologues, parmi lesquels Parsons (1951), ont montré à l'aide d'exemples, l'importance des processus de socialisation au cours desquels certaines normes sont progressivement intériorisées par les individus qui se sentent engagés à s’y conformer en dehors de tout calcul rationnel ou souci de sanction. Dans cet esprit, il est peu probable que le praticien observe les usages de sa profession par simple respect de la législation comptable. Celle-ci ne dispose pas de la pleine capacité à créer des conventions; le plus souvent, elle les entérine et en informe l'existence. L'ensemble des règles comptables existait bien avant que les textes ne les ratifient. En revanche, le regard et la considération de ses pairs constituent un garde-fou autrement plus efficace que toute instance judiciaire. Weber (1956, p. 68) en était convaincu : il y a convention «lorsque sa validité est garantie extérieurement par la chance que, si on s'en écarte à l'intérieur d'un groupe d'hommes déterminé, on s'expose à une réprobation 
(relativement) générale et pratiquement perceptible » en dehors de tout dispositif de coercition créé à cet effet. Sur ce sujet, le lecteur pourra s'étonner qu'il soit fait ici référence à l'analyse Wéberienne alors que l'axiomatique de Lewis fut mobilisée dans la section précédente. Il est vrai que certains économistes (Batifoulier, Larquier (de), 2001) ont opposé l'approche des deux auteurs attendu que le second avance comme seule sanction «l'échec de la coordination » alors que Weber considère la sanction comme intrinsèque à la convention en évoquant même la notion de "boycott social ». A notre sens, il s'agit plus d'un problème d'interprétation que d'une véritable opposition. Si Lewis n'exprime pas formellement l'idée de sanction mais de coordination manquée, rien n'exclut dans son propos la réprobation consécutive des autres convenants suite à cet échec. Sachant que l'absence de coordination affecte l'utilité des agents, il serait naturel qu'il en résulte des réactions d'hostilité. Au demeurant, on pourrait même lire dans la troisième proposition de son axiomatique " chacun préfère une conformité générale... ", le corollaire de la préférence à savoir une aversion à la déviance et un rejet du déviant.

- Mais le comptable s'y soumet volontairement ; typiquement, le professionnel qui pénètre dans un système de conventions ne se voit plus comme l'auteur de ses actes mais plutôt comme l'agent exécutif de leurs propres prescriptions. Il s'agit là d'un état de soumission se traduisant par un repli de l'individu derrière les pratiques conventionnelles, celles adoptées par ses pairs sur un territoire particulier. La pratique est à ce point institutionnalisée que même la question de sa rationalité, de sa légitimité, voire de sa licéité ne se pose plus.

- Les conventions comptables évoluent, elles ne sont pas figées. La convention n’est qu'un équilibre mimétique (Gomez, 1994) ; aussi, les conventions comptables établies sont parfois mises en doute par des conventions adverses dites "alternatives », et dont la cohérence et la pertinence du discours peuvent selon les conditions séduire la population des convenants. Diverses réactions sont envisageables en aval. La convention en place peut s'adapter en recadrant son discours ou en acceptant de partager son territoire avec une autre convention. Elle peut aussi, selon le contexte, affronter l'adversité en s'engageant dans une lutte qui pourra la renforcer ou bien la renverser en consacrant l'alternative au rang de convention nouvelle (Amblard, 2001).

Résumons-nous. L'incertitude qui entoure la qualification et l'enregistrement d'un événement est résolue parce qu'il existe un dispositif cognitif appelé «convention comptable » permettant de coordonner les actes du praticien. Cette convention qui évolue au sein d'un espace suppose une adhésion généralisée de la population concernée. La comptabilité apparaît ainsi comme un système composé d'un ensemble de conventions aussi nombreuses que le sont les problèmes liés à la modélisation comptable.

\section{Conclusion}

L'approche conventionnaliste nous a fourni de nouveaux outils pour analyser le modèle comptable et les acteurs qui l'animent. D'un abord plus sociologique, elle nous a entraîné à « extraire » l'acte du praticien de son isolement pour le considérer comme une procédure collective identifiable par sa morphologie comme toute autre convention.

La théorie postule en effet un individu socialisé. Ses actes au sein de l'espace comptable sont compréhensibles pour peu qu'on ait préalablement mis au jour les conventions auxquelles il fait référence pour agir. Il ne s'agit pas à proprement parler d'un message précis, formalisé et 
émanant d'une source unique, mais plutôt d'une somme d'informations convergentes, allant des textes officiels ayant force de loi aux prescriptions locales méconnues, et qui ensemble, participent à la définition des comportements attendus.

Les conventions ont aussi pour fonction d'organiser une " mise en scène " de l'information, destinée à rassurer l'utilisateur ; en procurant au modèle une cohérence dans la représentation du réel, elles apportent au professionnel une solution adaptée et surtout reconnue par la communauté à défaut d'être juste. Mais en a-t-il conscience? Qu'il s'agisse de conventions d'observation, de mesure, de réalisation ou de procédure, notre enquête a révélé que les conventions comptables sont à ce point prégnantes qu'elles agissent comme une programmation mentale collective donnant aux agents l'illusion d'être souverain ou que la solution s'impose d'elle-même en l'absence d'alternative. Difficile pourtant d'admettre leur neutralité. Les conventions résultent de choix qui modèlent considérablement l'image comptable et dans le même temps, entraînent discrètement le praticien et plus généralement l'utilisateur dans un schéma de pensée très orienté. Le reconnaître, c'est accepter que notre modèle comptable n'est qu'une construction contingente des groupes sociaux qui, historiquement, ont su établir par leur poids une vision conventionnelle de la firme. La représentation comptable n'est donc pas donnée mais bien convenue.

Pour autant, l'approche conventionnaliste ne se contente pas de jeter un regard renouvelé sur le modèle comptable ; elle l'enrichit en mettant l'accent sur des mécanismes sociaux profonds qui échappent à l'interprétation contractualiste largement prédominante dans notre champ d'étude. La théorie des conventions devrait alors nous permettre de mieux appréhender par quels processus les comportements se normalisent. Les récents scandales qui ont placé la comptabilité d'entreprise au cœur du débat économique montrent à quel point il devient primordial de comprendre comment se créent, évoluent et disparaissent les normes. Nous savons qu'elles ne sont pas figées ; l'histoire a montré qu'elles s’inscrivent, bien au contraire, dans un processus d'évolution inéluctable. Or, le changement dans les comportements collectifs, notamment les pratiques comptables, ne relève pas du pur hasard, loin s'en faut. La transformation progressive des conventions comptables répond à des influences et des courants profonds, s'appuyant sur des mécanismes collectifs et socialement construits ; la théorie des conventions devrait alors nous permettre d'en pénétrer plus efficacement la logique. 


\section{ANNEXE \\ PRESENTATION SOMMAIRE DE L'ENQUETE}

\section{- Choix de la population}

Le principal objet de cette enquête repose sur l'appréciation et l'analyse conventionnaliste des règles comptables. Nous avons alors décidé d’interroger 192 individus fortement impliqués dans la pratique de la comptabilité. Trois catégories de professionnels ont été retenues : les experts-comptables (106), les assistants de cabinet (45) et les comptables d'entreprises (41).

\section{- Fondement du questionnaire}

Notre principal objectif fut de vérifier le caractère conventionnel des règles comptables. La convention ne peut être un concept «fourre-tout»: tout n'est pas convention. Aussi, conformément à l'axiomatique de Lewis, référence en la matière, les questions furent alors sélectionnées en fonction de leur capacité à vérifier les cinq propositions de l'approche conventionnaliste. Elles furent aussi choisies en fonction de leur aptitude à apprécier le degré de prégnance des conventions au sein de la pratique. Nous souhaitions qu'elles apportent des éléments de réponses quant au rapport que le professionnel entretient avec elles: est-il conscient de leur caractère consensuel et alternatif, ou bien perd-il à leur égard tout ou partie de son sens critique au point d'en devenir un agent?

\section{- Les moyens utilisés}

La majeur partie des questions appelait des réponses binaires " oui / non »; toutefois, et cela fut riche d'enseignements, nous n'avons pas manqué de recueillir des commentaires, des réflexions sur l'avis et l'attitude des répondants. Il est évident que le caractère psychologique et social de l'objet du sondage peut difficilement se prêter à une analyse unique des pourcentages obtenus.

\section{- Questionnaire}

\begin{tabular}{|c|l|l|l|l|}
\hline Questions & Oui & Non & S.O & Réponses ouvertes \\
\hline $\begin{array}{c}\text { Globalement, pensez-vous que la pratique de la } \\
\text { comptabilité au sein de votre cabinet (ou entreprise) } \\
\text { est conforme aux usages de la profession? }\end{array}$ & & & & \\
\hline $\begin{array}{c}\text { Quel est votre sentiment à l'égard de vos confrères : } \\
\text { pensez-vous qu'eux aussi se conforment aux usages de } \\
\text { la profession? }\end{array}$ & & & & \\
\hline $\begin{array}{c}\text { Pensez-vous que vos confrères partagent votre sentiment? } \\
\text { En d'autres termes, pensez-vous que les autres } \\
\text { praticiens prêtent à leurs homologues la même } \\
\text { conformité aux usages de la profession ? }\end{array}$ & & & & \\
\hline $\begin{array}{c}\text { Préférez-vous que tous s'y conforment dans le cadre d'une } \\
\text { pratique homogène (oui) ou bien êtes-vous indifférent } \\
\text { à une multiplication des déviances qui aboutirait à une } \\
\text { pratique hétérogène ? (non) }\end{array}$ & & & & \\
\hline $\begin{array}{c}\text { Selon vous, comment les autres praticiens interrogés ont-ils } \\
\text { répondu à la question précédente ? } \\
\text { même réponse (oui) } \\
\text { autre réponse (non) }\end{array}$ & & & & \\
\hline $\begin{array}{c}\text { Pour quelles raisons, d'après vous, les salariés ne sont-ils } \\
\text { pas inscrits au bilan? }\end{array}$ & & & & \\
\hline Cela vous paraît-il logique? & & & & \\
\hline
\end{tabular}

\footnotetext{
${ }^{2}$ Sans opinion, ne sait pas répondre ou réponse très hésitante.
} 
Selon vous, existe-t-il un texte en la matière ?

Le connaissez-vous?

Pour ceux qui ont répondu oui à la question 7 : pour quelles raisons les clubs de football inscrivent-ils alors leurs joueurs à l'actif?

Que dire alors de certains dirigeants ou précieux collaborateurs dont les contrats se négocient entre firmes (pour ceux qui ont trouvé une justification à la question 10)?

La rémunération des fonds apportés par le banquier est considérée comme une charge, celle des fonds apportés par l'actionnaire comme un prélèvement sur les bénéfices. Cela vous paraît-il logique ?

Pourrait-on concevoir une autre façon d'enregistrer les dividendes versés ?

Connaissez-vous les raisons pour lesquelles les biens financés par crédit-bail ne figurent pas à l'actif ?

Cela vous paraît-il logique ?

Y aurait-il, selon vous un critère d'inclusion autre que la propriété juridique?

Si oui, pensez-vous qu'on s'y dirige?

A l'égard du crédit-bail, la règle d'enregistrement est-elle la même pour les comptes consolidés ?

Pour quelles raisons, les comptes consolidés ont-ils prévu un autre mode de comptabilisation?

Une entreprise fait l'acquisition d'un ordinateur à crédit assortie d'une clause de réserve de propriété, doit-elle l'inscrire à l'actif du bilan?

Y aurait-il une autre façon de procéder?

L'enregistrement actuel vous paraît-il logique?

N’y a-t-il pas contradiction avec l'enregistrement du créditbail ?

Pratiquez-vous l'amortissement dégressif?

- 24-1 - jamais

- 24-2 - rarement

- 24-3 - oui dès que cela est possible

Si jamais ou rarement pourquoi ?

Si oui enregistrez-vous l'amortissement dérogatoire lorsqu'il n’y a pas de correspondance entre l'amortissement dégressif et l'amortissement économiquement justifié ?

Si non pourquoi puisque les textes le prévoient?

Pensez-vous qu'à ce sujet les autres cabinets procèdent de la même manière ?

Le cas échéant, enregistrez-vous les écarts de conversion en fin d'exercice?

Si non pourquoi, puisque les textes le prévoient?

A votre avis, existerait-il dans l'absolu une autre façon d'enregistrer les écarts de conversion?

Le principe des coûts historiques (valeur d'origine) vous paraît-il cohérent?

Existe-t-il, selon vous, d'autres modes d'évaluation?

Connaissez-vous les principes comptables mentionnés dans le PCG ?

35. Si oui, y avez-vous parfois recours pour résoudre un problème d'enregistrement? 


\section{Notes}

1. La question paraît d'autant plus décalée que la comptabilité fut traitée pendant fort longtemps comme une simple technique arithmétique au service d'une corporation particulière et dépourvue d’intérêt scientifique. Son enseignement dans les universités françaises est somme toute assez récent et plus encore l'intérêt que lui portent certains chercheurs.

2. Il est à noter que la frontière séparant le patrimoine de l'entreprise et celui de son propriétaire n’est toujours pas clairement définie.

\section{Bibliographie}

AMBLARD M. (2001), Comptabilité et conventions, L’Harmattan, Paris.

AMBLARD M. (2003), "Vers une théorie sur la dynamique des conventions" in AMBLARD M. (Ed), Conventions et management, De Boeck, Louvain, Belgique, pp. 139158.

BATIFOULIER P., Larquier (de) G. (2001), " De la convention et de ses usages », in BATIFOULIER P. (dir), Théorie des conventions, Economica, Paris, pp. 9-31.

BELKAOUI A. (1985), Théorie comptable, Presse de l’Université du Québec.

BOLTANSKI L. et THÉVENOT L. (1983), Les économies de la grandeur, Cahiers du centre d'études de l'emploi, Presse Universitaire de France, Paris.

CAPRON M. (1990), «La comptabilité : faut-il y croire pour avoir confiance ? », Gérer et comprendre, décembre, pp. 75-83.

COLASSE B. (1996), "Commentaire analytique et critique du projet de cadre conceptuel du CDPC », Revue française de comptabilité, $n^{\circ} 282$, octobre, pp. 29-34.

DELEUZE G. (1988), Nietzsche et la philosophie, Presse Universitaire de France, Paris.

DETCHESSAHAR M. (2003), " Pour un relâchement de l'axiomatique conventionnaliste en sciences de gestion - Réflexion à partir d'une étude de cas ", in AMBLARD M. (Ed), Conventions et management, De Boeck, Louvain, Belgique, pp. 203-230.

DUMOUCHEL P. (1992), "L'évolution des règles et des conventions ", Cahiers d'épistémologie, n 179, Université du Québec, Montréal, pp. 1-22.

FAVEREAU O. (1989), " Marchés internes, marchés externes », Revue économique, n², mars, pp. 276-325.

GENSSE P. (1995), «L'invention comptable de la réalité : entre la règle et le mythe » in Mélanges en l'honneur du professeur Claude PÉROCHON, Foucher, Paris, pp. 221-231.

GENSSE P. (2003), "Introduction générale » in AMBLARD M. (Ed), Conventions et management, De Boeck, Louvain, Belgique, pp. 13-24.

GOFFMAN E. (1973), La mise en scène de la vie quotidienne, Les Editions de Minuit, Paris.

GOMEZ P.Y. (1994), Qualité et théorie des conventions, Economica, Paris.

LASSÈGUE P. (1993), Lexique de comptabilité, Dalloz, $3{ }^{\text {ème }}$ édition, Paris.

MERCIER E. (2003), " Pour une lecture conventionnaliste du changement organisationnel : le cas d'une entreprise de service public (la RATP) » in AMBLARD M. (Ed), Conventions et management, De Boeck, Louvain, Belgique, pp. 179-202.

MILGRAM S. (1986), Soumission à l’autorité, Calmann-Lévy, Paris.

ORLÉAN A. (1989) "Pour une approche cognitive des conventions économiques », Revue Economique $\mathrm{n}^{\circ} 2$, mars, pp. 241-272.

ORLÉAN A. (1999), Le pouvoir de la finance, Odile Jacob, Paris.

PARSONS T. (1951), The Social System, Glencoe.

SCHELLING T. (1960), The strategic of conflict, Harvard University Press. 
WEBER M. (1956), Economie et société, tome 1, Agora, Paris, 2003, traduction de Wirtschaft und Gesellschaft, Tübingen, Mohr, 1956. 